\title{
Clinical governance in primary care Improving quality in the changing world of primary care
}

\author{
Rebecca Rosen
}

Publication of A First Class Service placed quality improvement at the centre of the health policy stage. ${ }^{1}$ The term clinical governance was used to capture the range of activities required to improve the quality of health services. Central among these were the need for all NHS organisations to develop processes for continuously monitoring and improving the quality of health care and to develop systems of accountability for the quality of care that they provided.

Evidence based practice, audit, risk management, mechanisms to monitor the outcomes of care, lifelong learning among clinicians, and systems for managing poor performance will all contribute to the development of effective clinical governance. In addition, the term combines an emphasis on improving care for individual patients with quality improvement targeted at whole populations. This wide ranging approach creates a challenge for those leading the implementation of clinical governance.

Primary care groups and trusts are the organisations through which clinical governance will be developed at a local level and local priorities identified. Yet the nitty-gritty work of clinical governance will be undertaken by the members of individual practices and primary care teams. The articles in this series explore the meaning of clinical governance in primary care. They focus on the roles of primary care groups and trusts; the knowledge, skills, and support needed by individual practitioners; and the emerging requirements for accountability between practitioners, the public, and the wider NHS hierarchy.

This article examines definitions of quality, the values underlying them, and early approaches to implementing clinical governance in primary care. Without consensus on these issues it will be difficult to develop a shared view of what clinical governance is and of how to implement it.

Recognising the fragmented organisational structure of primary care and the traditional independence of general practitioners, the second article in the series will focus on accountability for quality. The need for organisational and professional development to facilitate changes in the culture of primary care, and improvements in clinical knowledge, skills, and leadership, will be covered in the third and fourth articles respectively. The final article will consider the extensive range of knowledge and information required for the successful implementation of clinical governance.

\section{Building on established foundations}

The work required for effective clinical governance is not new. The literature about quality in general practice is well established and its focus is expanding to encompass the wider primary care team. General practitioners, community nurses, and other primary care practitioners have been involved in audit and guideline development for years, and the 1998 report of the chief

\section{Summary points}

Quality improvement systems for general practice exist, but there have been few attempts to involve all primary care team members in quality improvement work

Clinical governance in primary care must focus on individual patients and whole populations; this creates tensions between a view of good practice based on individual rights and a population approach focused on fair distribution of services

Primary care services are fragmented across multiple providers, with no clear managerial or professional hierarchy through which to implement clinical governance

Clinical governance in primary care must build on and develop existing activities to improve the quality of services

medical officer heralded a move from continuing medical education focused on general practitioners to continuing professional development involving the whole practice team. ${ }^{2}$ Moreover, such work is not confined to the United Kingdom, and there is potential to learn lessons from abroad (box).

Although the different strands of quality improvement work based in the United Kingdom represent a good foundation for clinical governance, their implementation has been patchy, with no obligation on practitioners to become involved. A major challenge

International examples of quality improvement work in primary care

Peer review and individual feedback on clinical performance (New Zealand)-Voluntary membership of independent practitioners' associations in which aspects of clinical practice by participating general practitioners are reviewed through peer discussion groups with a view to reducing expenditure $^{3}$

Lifelong learning (Germany)-Diploma run by state medical association for fully trained doctors with five years' experience. Based on 200 hours of teaching covering guidelines and best practice, health economics, and quality management ${ }^{4}$

Revalidation of doctors (Norway)-Recertification every five years, based on 200 hours of continuing medical education and three months' work in a hospital. Linked to a $10-15 \%$ pay increase 5

Public involvement in medical regulation and accountability (Canada)-Lay representation on governing councils of medical colleges, registration committees, and complaints and disciplinary committees

Technology assessment programmes to promote effective practice (UK, Spain, Netherlands, and other countries)-Well established technology assessment programmes linked to dissemination of findings for use in guideline development ${ }^{7}$ 
for the lead clinicians responsible for clinical governance will be to involve all members of the primary care team in such work. It will be important to learn from and build on existing initiatives and to avoid reinventing quality improvement wheels. However, developing clinical governance in primary care is complicated by the diverse and evolving nature of primary care services.

\section{Understanding the context}

It is only in the past decade or so that consideration of the "primary care team" has begun to replace narrower discussions about general practice. There is no single definition of the scope or constituency of primary care, however, and the term has often been used interchangeably with general practice in policy papers. ${ }^{89}$ Much existing work on quality therefore focuses on general practice, linking the meaning of quality to perceptions about the aims and values of general practice and the perspective from which questions about quality are asked.

Toon's conceptual examination of "good general practice" ${ }^{" 10}$ distinguishes three different approaches-a disease focused, biomedical model; a patient focused, humanist model; and a population focused, public health model. Toon's argument that the meaning of good general practice differs between models goes some way to explaining the variety of approaches to assessing and improving quality which have been put forward (see box below).

Howie et al have attempted to assess quality from the patients' perspective by measuring the extent to which their consultation enables them to understand

Recent documents on improving quality in general practice and primary care groups

Established mechanisms and models for assessing and improving quality in primary care

- Royal College of General Practitioners membership and fellowship by assessment ${ }^{20}{ }^{21}$-Process of formal peer review leading to membership or fellowship of the college. Involves assessment of medical records, staff policies, audits, inspection, video assessment

- Roland et al. Quality assessment for general practice; supporting clinical governance in PCGs. $(1998)^{13}-14$ key indicators reflecting timely access to care, good clinical care, and good interpersonal care

- Roland and Baker. Clinical governance: a practical guide for primary care teams. (1999) ${ }^{22}$-Practical guide to the activities of clinical governance and how to implement them within practices and primary care teams

- King's Fund Health Quality Service. Primary care group standards. $(1999)^{23}$-Practical guidance on establishing primary care groups, including developing their clinical governance role

- McColl et al. Performance indicators for primary care groups. $(1998)^{12}$-Academic paper on potential of evidence based performance indicators for primary care groups

- Baker et al. Model for clinical governance in primary care groups. $(1999)^{24}-$ Practical model linking quality standards to systems of accountability and mechanisms for monitoring practice

Conceptual discussions of the meaning of quality

- Toon. What is good general practice? (1994) -Quality as a combination of empirical fact and value judgments. Three models of general practice, each reflecting a different balance between empirical and value laden practice

- Greenhalgh and Eversley. Quality in general practice. (1999) ${ }^{14}$-Identify patient, clinician, and organisational aspects of quality in general practice and develop matrix approach to identifying quality improvement priorities

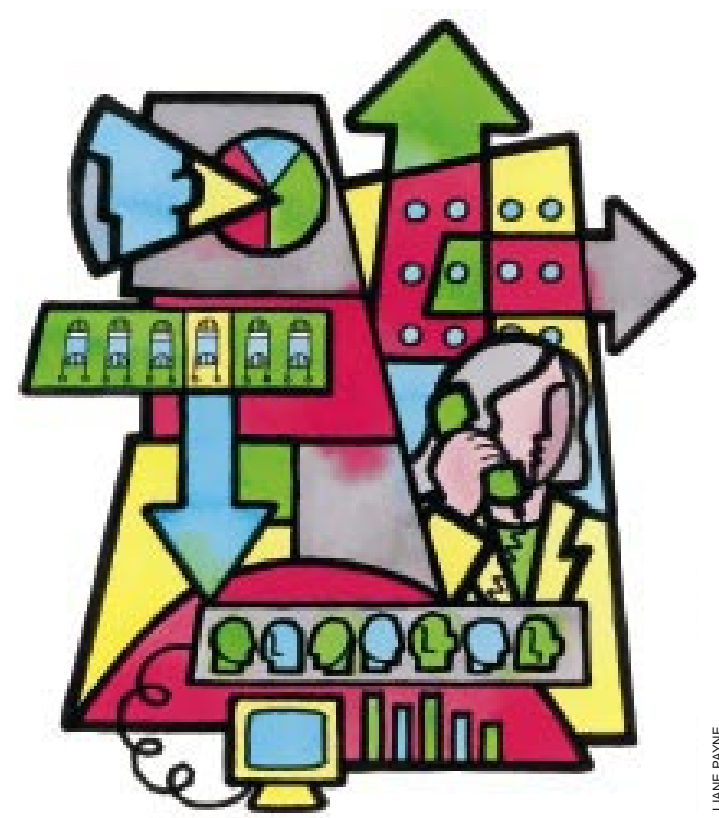

and cope with their illness. ${ }^{11}$ McColl et al take a more biomedical approach, proposing that primary care groups can assess the potential impact of different clinical activities by applying evidence on clinical effectiveness to their own practice population. ${ }^{12}$

Roland et al combine patient, population, and organisational perspectives to identify 14 markers of quality in general practice. ${ }^{13}$ These include measures of access and availability (for example, waiting times and telephone access to doctors); clinical markers relating to the quality of acute, chronic, terminal, and preventive care; and measures of prescribing and referrals. Greenhalgh and Eversely explore several different perspectives from which to consider quality in general practice. ${ }^{14}$ They distinguish patient, activity and performance, evidence based, educational, and managerial perspectives, each of which focuses on a different cluster of markers of quality.

Greenhalgh and Eversley argue that no single perspective can be used to describe the quality of care in practice. In a similar vein, Toon argues that it is impossible to define a single version of good quality general practice if there is no agreement on what it is aiming to do and the values pursued through it. ${ }^{10}$ These points are of relevance to primary care groups and trusts whose key tasks are developing primary care services, commissioning health care, and improving the health of the local population. This combination of individual and population based goals needs to be reflected in the range of clinical governance work undertaken.

\section{Values underpinning clinical governance}

Explicit consideration of the values which underpin work on clinical governance may help to avoid conflicts and increase the clarity of the aims during implementation. Fugelli and Heath have discussed the conflict between individual rights and utilitarian ethics which arises when modern general practice (and now primary care groups and trusts) combine a commitment to individual patients with population based, public health type goals. ${ }^{15}$ New has argued that such 
tensions are inevitable in the NHS: the main challenge for health professionals is to promote debate on which values should prevail in any particular situation. ${ }^{16}$ If, for example, a primary care group chooses diabetes as a priority area it will still need to decide between individually focused priorities such as implementing evidence based treatment protocols and population goals such as reducing inequalities in access and use by different groups.

A second example of these conflicts relates to guideline implementation, which is a core part of clinical governance work. Most guidelines aim to promote best clinical practice, but some-such as the recent guidance on prescribing of Viagra ${ }^{17}$ - can be seen as rationing tools which exclude selected patients from treatment. Willingness to implement such guidance will in part be shaped by the balance that primary care groups and trusts seek between improving care for individuals and for populations.

A statement of more general values will also be important in implementing clinical governance. One London primary care group, for example, has incorporated a statement of values and principles into its clinical governance business case, including commitments to reflective learning as opposed to a blame culture and clinician led multidisciplinary team work. ${ }^{18}$ This type of explicit statement should help to make peer review and work with poorly performing clinicians less threatening and more productive.

\section{Implementing clinical governance in primary care}

Early central guidance on clinical governance defined a series of tasks to be undertaken: establishing leadership, accountability, and working relationships; conducting a baseline assessment of capacity and capability; formulating a clinical governance development plan; and clarifying reporting arrangements for clinical governance. ${ }^{19}$ In addition, various authors have produced guidance and theoretical models for implementing clinical governance (box).

Baker et al have emphasised the intimate link between quality improvement and accountability. ${ }^{24}$ Standards for quality improvement, associated systems of accountability, and mechanisms for achieving change are defined at the level of individual healthcare professionals (professional codes of practice, continuing professional development, etc) practice teams (implementation of health improvement programme priorities or local guidelines) and primary care groups (implementing guidance from the National Institute for Clinical Excellence and national service framework recommendations).

The Royal College of General Practitioners has proposed a different framework with three core goals: protecting patients, developing individual practitioners, and developing teams and systems for quality improvement. ${ }^{25}$ In contrast to this focus on individuals and teams, Scally and Donaldson emphasised the need for organisational development-particularly clinical leadership and team working. ${ }^{26}$ This is particularly relevant in the context of the multiple independent practitioners now clustered into primary care groups and trusts.
On the ground, primary care groups and trusts are taking many different approaches to clinical governance. ${ }^{27}{ }^{28}$ Some are using work on a single clinical issue (for example, auditing prescribing for heart disease or developing practice registers for diabetes) to develop interest and involvement among local clinicians. Others see clinical governance as underpinning all their work, linking it to health improvement and primary care investment plans. Some groups and trusts are taking a lead in identifying priorities; others are encouraging individual practices to select areas for quality improvement. Some are working to establish common information technology and data collection systems to monitor local services, but others have not yet turned their attention to this. ${ }^{27} 28$

Despite this variation, there is some common ground. A survey of primary care groups by the Audit Commission shows that $70 \%$ will be undertaking interpractice audits and working to improve data for monitoring the quality of care. ${ }^{29}$ Three quarters were able to call on support from external agencies such as public health departments and medical audit groups. A similar proportion have now set targets for accountability, many of which relate to prescribing.

Despite this promising start, another survey of clinical governance leaders revealed concerns about implementation at a local level. Foremost among these were the lack of resources for the job, the size of the workload created by clinical governance, and the difficulties of moving primary care professionals towards the open and participative culture that is required. ${ }^{30}$

Other factors may complicate the development of clinical governance in primary care. A legacy of domination by general practitioners will make it hard to develop the necessary multiprofessional team working. The fragmentation of primary care across multiple small providers means that there is no clear professional or managerial hierarchy through which to drive implementation. Given the monitoring of services that is also required for successful clinical governance, the embryonic state of information technology in primary care-particularly in singlehanded practices-will limit the ability of primary care groups and trusts to monitor outcomes.

\section{The research agenda for clinical governance}

Much is already known about promoting effective practice and changing clinical behaviour in primary care ${ }^{31}{ }^{32}$ However, the introduction of clinical governance throws up many further research questions. What are effective mechanisms for developing accountability in primary care, and what incentives will increase participation in clinical governance? What are the advantages and disadvantages of different methods of public involvement in clinical governance? What are the most effective methods for education and training in multidisciplinary primary care teams and for managing underperforming colleagues?

Such studies will be methodologically challenging, requiring the integration of several academic disciplines in a way which produces valid and timely results. The forthcoming NHS research programme in service delivery and organisation provides an important 
opportunity to develop the knowledge required for effective clinical governance.

\section{Resources for clinical governance}

Though almost any proposed change to primary care services triggers calls for additional resources, it is clear that effective clinical governance is resource intensive. Support is needed for audit, particularly for singlehanded and small practices. Developing and implementing local guidelines requires input from clinicians, many of whom will require locum cover. Personal and professional development requires training and education that must be funded and for which locum cover must be provided. Additional information technology and information resources will be needed to monitor progress.

In the survey of clinical governance leaders in London mentioned above, $46 \%$ of respondents expressed concern about the limited time, staffing, and resources available for implementation. ${ }^{30}$ Some primary care groups and trusts are identifying in-house resources in terms of the skills and knowledge of individual members, and it will be essential not to exacerbate resource limitations by failing to harness these. There is a real danger that the tendency for NHS organisations to forget about or lose skills and knowledge during periods of change and reorganisation may repeat itself in relation to clinical governance.

It will take strong clinical leadership and imagination to develop clinical governance to its full potential. But without adequate resources to kick-start the process, the snowball of clinical governance will be slow to start rolling.

1 Department of Health. A first class service. London: Department of Health, 1998.

2 Chief Medical Officer. A review of continuing professional development in general practice. London: Department of Health, 1998. www.open.gov.uk/ $\mathrm{doh} / \mathrm{cmo} / \mathrm{cmoh} . \mathrm{htm}$ (accessed 21 July 2000 ).

3 Malcolm L, Mays N. New Zealand's independent practitioner associations: a working model of clinical governance in primary care? BMJ 1999;319:1340-2

4 Pugner K. Quality über alles? The German approach to clinical regulation. BrJ Health Care Manage 1998;12(suppl):4-7.

5 Grol R. Quality assurance in general practice: the state of the art in Europe. Fam Pract 1994;11:460-7

6 Salter B. Reforming the regulation of the medical profession:putting the UK into perspective. Norwich: University of East Anglia, 2000.
7 Banta HD, Battista R, Gellband H, Jonnson E, eds. Health care technology and its assessment in eight countries. Washington, DC: Office of Technology Assessment of US Congress, 1995.

8 Periera Gray D. Planning primary care. London: Royal College of Practitioners, 1992. (Occasional paper No 57.)

9 North West Thames Regional Office. Developing primary health care: $a$ direction statement.London: North West Thames Regional Office, 1993.

10 Toon P. What is good general practice? London: Royal College of General Practitioners, 1994

11 Howie JG, Heaney DJ, Maxwell M. Measuring quality in general practice. Pilot study of needs, process and outcome measure. London: Royal College of General Practitioners, 1997.

12 McColl A, Roderick P, Gabbay J, Smith H, Moore M. Performance indicators for primary care groups: an evidence based approach. BMJ 1998:317:1354-60.

13 Roland M, Holden J, Campbell S. Quality assessment for general practice; supporting clinical governance in PCGs. Manchester: National Primary Care Research and Development Centre, University of Manchester, 1998. www.npcrdc.man.ac.uk/Pages/Publications/PDF/Quality.pdf (accessed 7 August 2000).

14 Greenhalgh T, Eversley J. Quality in general practice. London: King's Fund, 1999.

15 Fugelli P, Heath I. The nature of general practice. BMJ 1996;312:456-7.

16 New B. A good enough service:values tradeoffs and the NHS. London: King's Fund, 1999.

17 Ferriman A. UK government finalises restrictions on Viagra prescribing. BMJ 1999;318:1305.

18 Harrow West Primary Care Group. Clinical governance/education and training sub-committee business plan 1999-2000. London: Harrow West PCG, 1999.

19 NHS Executive. Clinical governance in the new NHS. Leeds, NHS Executive, 1999. (HSC1999/065.)

20 Royal College of General Practitioners. Membership by assessment of performance. Guidance notes for applicants. London: RCGP, 1999.

21 Periera Gray D. Fellowship by assessment.London: RCGP, 1997. (Discussion paper No 50; revised edition including 1995-6 guidance.)

22 Roland M, Baker R. Clinical governance: a practical guide for primary care teams. Manchester: National Primary Care Research and Development Centre; Leicester: Clincal Governance Research and Development Unit, 1999.

23 King's Fund, Health Quality Service. Primary care group standards. London: King's Fund, HQS, 1999.

24 Baker R, Likhani M, Fraser R, Cheater F. A model for clinical governance in primary care groups. BMJ 1999;318:779-83.

25 Royal College of General Practitioners. Clinical governance:practical advice for primary care in England and Wales. London: RCGP, 1999.

26 Scally G, Donaldson L. Looking forward: clinical governance and the drive for quality improvement in the new NHS in England. BMJ 1998;317:61-5.

27 Wilkin D, Gillam S, Leese B. The national tracker survey of primary care groups and trusts: progress and challenges 1999-2000. Manchester: National Primary Care Reserch and Development Centre and the King's Fund, 2000 .

28 Wye L, Rosen R, Dewar S. Clinical governance in primary care: a review of baseline assessments. London: King's Fund, 2000.

29 Audit Commission. The PCG agenda: early progress of PCGs in 'The New NHS'. London: Audit Commission, 2000.

30 Hayward J. Rosen R, Dewar S. Clinical governance: thin on the ground. Health Services 1999 August 26:26-7.

31 Getting evidence into practice. Effective Health Care 1999;5(1).

32 Wensing M, van der Weijeden T, Grol R. Implementing guidelines and innovations in general practice: which interventions are effective? $\mathrm{Br} \mathrm{J}$ Gen Pract 1998;48:991-7.

\section{A memorable interview \\ Experience not required}

It was 1957, and I had just completed my preregistration year. The plan was for me to join my eldest brother in general practice, but he insisted that I first did a six month obstetric post. I applied for various posts and was interviewed here and there, all without success. In due course I applied and was interviewed for a post at what was then Paddington General Hospital in the Harrow Road. I sat rather forlornly with seven or eight other hopefuls in the waiting room and, one by one, we were summoned to the interview room and returned to wait.

Eventually, all had been interviewed and the personnel officer came out and asked one of the assembled group to go in again, adding that she was very sorry but the rest of us could leave. We waited a bit longer until the successful candidate came out smiling and went on his way. After a few moments, the personnel officer came into the waiting room once more, saying to the lingering two or three disappointed applicants, "By the way, there is a vacancy for a locum senior house officer in anaesthetics. Are any of you interested?"
At that time, I was married with two children and out of work. I was interested in anything. I expressed interest and was assured that experience in anaesthetics was not required. With a minimum of formality, I was offered the post for six months and thus began my training and subsequent 40 year career as an anaesthetist. I never did get an obstetric post or enter general practice and have enjoyed tremendously the career that fate selected for me.

John S M Zorab consultant anaesthetist emeritus, Bristol

We welcome articles of up to 600 words on topics such as A memorable patient, A paper that changed my practice, My most unfortunate mistake, or any other piece conveying instruction, pathos, or humour. If possible the article should be supplied on a disk. Permission is needed from the patient or a relative if an identifiable patient is referred to. We also welcome contributions for "Endpieces," consisting of quotations of up to 80 words (but most are considerably shorter) from any source, ancient or modern, which have appealed to the reader. 